\title{
Substrate-specific diffusion of select dicarboxylates through Chlamydia trachomatis PorB
}

\author{
Aya Kubo ${ }^{1}$ and Richard S. Stephens ${ }^{1,2}$
}

Author for correspondence: Richard S. Stephens. Tel: +1 510643 9900. Fax: +1 5106431537. e-mail: rss@uclink4.berkeley.edu

1 Division of Infectious Diseases, School of Public Health, 140 Earl Warren Hall, University of California, Berkeley, CA 94720, USA

2 Francis I. Proctor Foundation, University of California, San Francisco, CA 94143, USA

\begin{abstract}
Chlamydiae contain two porins, MOMP and PorB, that facilitate diffusion of solutes through the outer membrane. MOMP is a general porin that permits the diffusion of a wide variety of compounds including carbohydrates and amino acids. The relative inefficiency of PorB as a general porin and its low abundance in the outer membrane suggest that it may function as a substratespecific porin. The tricarboxylic acid (TCA) cycle of chlamydiae is incomplete and to function would require the exogenous acquisition of 2-oxoglutarate or glutamate. A liposome-swelling assay for anions as well as an enzyme-linked liposome assay were used to demonstrate the efficient diffusion of dicarboxylates such as $\mathbf{2 - o x o g l u t a r a t e}$ through PorB. These data demonstrate that PorB is a dicarboxylate-specific porin that may feed the chlamydial TCA cycle and provide chlamydiae with carbon and energy production intermediates.
\end{abstract}

Keywords: major outer-membrane protein, porin, 2-oxoglutarate

\section{INTRODUCTION}

The chlamydial genome is about four times smaller than the Escherichia coli genome. The small genome size of chlamydiae is attributed to the obligate intracellular biology of these organisms. Because Chlamydia spp. grow within host cells and are sensitive to amino acid limitations (Harper et al., 2000) and lack coding for essential metabolic pathways (Stephens et al., 1998), it is inferred that they have access to metabolites from the host cell. The genome encodes a large number of innermembrane transporters with broad substrate specificity and few genes for the biosynthesis of metabolites such as amino acids (Kalman et al., 1999; Stephens et al., 1998). Thus chlamydiae have a variety of inner-membrane transport systems for acquiring metabolic intermediates from host-cell pools.

Previous experimental results have supported the 'energy parasite' hypothesis that suggests that chlamydial growth depends on the acquisition of ATP and other high-energy metabolites from the host (Moulder, 1991). Interestingly, the genome sequence has

Abbreviations: MOMP, major outer-membrane protein; TCA cycle, tricarboxylic acid cycle. revealed that Chlamydia may have the potential to produce oxidative and substrate-level ATP. Chlamydiae have the enzymic machinery for the Embden-Meyerhof pathway, the pentose phosphate pathway and the tricarboxylic acid (TCA) cycle (Kalman et al., 1999; Stephens et al., 1998). However, according to predictions based upon the genome sequence, the TCA cycle is incomplete in Chlamydia and is apparently missing three enzymes: citrate synthase, aconitase and isocitrate dehydrogenase (Kalman et al., 1999; Stephens et al., 1998). The implication of the missing TCA cycle enzymes is that glutamate and 2-oxoglutarate are obtained from the host cell as carbon and energy substrates. If 2-oxoglutarate is available from the host cell, then the cycle may be initiated with 2-oxoglutarate as an initial substrate and ending with the production of oxaloacetate. Chlamydiae encode an orthologue of a dicarboxylate transporter (SodTi). This orthologue is most similar to a transporter in spinach chloroplast envelopes that transports 2-oxoglutarate into the chloroplast (Weber et al., 1995). Therefore, in Chlamydia this translocator could transport 2-oxoglutarate through the inner membrane, allowing for net carbon entry into the TCA pathway. Thus, it is predicted that chlamydiae obtain 2-oxoglutarate from the host cell. It has recently been shown in vitro that chlamydiae utilize glucose as the major source of carbon but that 2-oxoglutarate also 
serves to support chlamydial viability and growth (IliffeLee \& McClarty, 2000).

Chlamydia has a major outer-membrane protein (MOMP) that functions as a general porin (Bavoil et al., 1984; Jones et al., 2000; Wyllie et al., 1998). The genome has also revealed a number of predicted outer-membrane proteins that have not been previously identified in Chlamydia. We recently used the genome analysis to aid in the discovery of a putative porin (PorB). PorB is localized in the outer-membrane complex of Chlamydia and is surface accessible (Kubo \& Stephens, 2000). This protein has porin activity when tested in a liposomeswelling assay but is significantly less efficient in diffusion of polysaccharide and amino acid solutes than MOMP (Kubo \& Stephens, 2000). Relative to the preponderance of MOMP in chlamydial outer membranes, PorB is much less abundant (Kubo \& Stephens, 2000). Differences in general pore-forming activity, as well as differences in the amount present in the chlamydial outer membrane, suggest a unique role for each of the porins. The presence of PorB in small amounts is difficult to understand unless PorB has a role as a substrate-specific porin that is efficient in the selective transport of particular classes of molecules. RT-PCR analysis and cell staining at various time points indicated that this protein is expressed throughout the developmental cycle (Kubo \& Stephens, 2000). Thus PorB expression is not differentially regulated and is likely not a secondary general porin that serves an analogous purpose as MOMP, being upregulated under different growth conditions.

We sought to determine if PorB is a substrate-specific porin that preferentially facilitates the translocation of certain classes of substrates into the periplasmic space. Our approach was to use the information derived from the chlamydial genome to aid in the identification of metabolites that are predicted to be obtained from the host and determine if these metabolites enter through PorB and/or MOMP.

\section{METHODS}

Chemicals. Chemicals used for this study were purchased from Sigma.

Expression and purification of PorB and MOMP. PorB was cloned and expressed in the E. coli TOP10 (Invitrogen), as previously described (Kubo \& Stephens, 2000). The protein was isolated from E. coli outer membranes using octylglucoside by previously described methods (Kubo \& Stephens, 2000). Briefly, E. coli containing the PorB gene was grown until the cultures reached an $\mathrm{OD}_{600}$ of $0 \cdot 6$ and arabinose was added to induce the expression of PorB. PorB was cloned with a C-terminal His tag and was purified by nickel column chromatography using the His Bind Purification system (Novagen) after removing the octylglucoside by dialysis using PBS and then $1 \times$ BIND buffer (Novagen).

The synthetic gene encoding MOMP (ompA) was constructed in E. coli HMS 174 (DE3) and has been previously described (Jones et al., 2000). E. coli HMS 174 (DE3) without the plasmid was used as a control strain. The outer membranes of
E. coli expressing MOMP and E. coli not expressing MOMP were isolated using the method of Osborn \& Munson (1974) with a few previously described modifications (Jones et al., 2000). Purified protein and outer membranes for use in the liposome-swelling assay were quantitated according to the Lowry method.

Liposome-swelling assay. This assay was performed according to the method of Nikaido \& Rosenberg (1983) with the following modifications: (1) liposomes were made by mixing $5 \cdot 0 \mu \mathrm{mol}$ phosphatidylcholine and $0 \cdot 02 \mu \mathrm{mol}$ dicetyl phosphate with PorB or outer-membrane proteins to $\mathrm{OD}_{400}$ readings between 0.4 and 0.7 and (2) the liposome drying time was longer than $2 \mathrm{~min}(5 \mathrm{~min})$, but at a lower temperature of $37^{\circ} \mathrm{C}$. Liposomes were made with dextran T-40 $(15 \%$ dextran $\mathrm{T}-40$ in $5 \mathrm{mM}$ Tris $/ \mathrm{HCl}, \mathrm{pH} 7 \cdot 5)$ inside. Since stachyose is impermeable to the porins, it was used as a control to determine the iso-osmotic concentration of other solutes. The concentration of stachyose that produced no swelling or shrinking of the proteoliposomes was determined to be the iso-osmotic concentration. The swelling rates were determined as $\mathrm{d}\left(1 / \mathrm{OD}_{400}\right) / \mathrm{d} t$ from the optical-density changes between 10 and $20 \mathrm{~s}$ (Nikaido \& Rosenberg, 1983).

Liposome-swelling assay for testing anions. Liposomes were made according to the method described above with a few modifications. The following were added to phosphatidylcholine and dicetyl phosphate dried with PorB $(6 \mu \mathrm{g}): 4 \mathrm{mM}$ $\mathrm{NAD}^{+}, 12 \mathrm{mM}$ stachyose, $1 \mathrm{mM}$ imidazole-NAD buffer $(\mathrm{pH}$ $6 \cdot 0)$. The test solution consisted of $1 \mathrm{mM}$ imidazole-NAD ( $\mathrm{pH}$ 6.0), $1 \mathrm{mM}$ sodium-NAD and $6 \mathrm{mM}$ disodium salt of the anion to be tested (2-oxoglutarate, succinate, oxaloacetate, malate or citrate). Control liposomes without protein were used to determine the isotonic concentration of the test solutions.

Enzyme-linked liposome-swelling assay. Liposomes were made as described above with addition of $50 \mathrm{mM}$ potassium phosphate, $2.5 \mathrm{mM} \mathrm{NAD}{ }^{+}, 0.2 \mathrm{mM}$ thiamin pyrophosphate, $1.0 \mathrm{mM}$ magnesium chloride, $0.13 \mathrm{mM}$ coenzyme A, $2.6 \mathrm{mM}$ cysteine and $5 \cdot 0$ units 2-oxoglutarate dehydrogenase. Various concentrations of 2-oxoglutarate $(0 \cdot 001-1 \mathrm{mM})$ were used as test solutes. Liposomes containing PorB $(6 \mu \mathrm{g})$ and control liposome without protein were made with the reaction mixture, washed through a Sephadex column (S-300) equilibrated with reaction mixture without 2 -oxoglutarate dehydrogenase, and placed inside a cuvette. 2-Oxoglutarate was added to the reaction and mixed. The formation of NADH was measured by the increase in $A_{340}$.

\section{RESULTS}

\section{Permeability of solutes through PorB}

The liposome-swelling assay was used to test poreforming activity of PorB. This assay was used because it is well established and because it gives precise information on the rates of diffusion of solutes through the porin channels (Nikaido \& Rosenberg, 1983). PorB was purified by nickel column chromatography and incorporated into liposomes as described previously (Kubo \& Stephens, 2000). Liposomes enriched for MOMP were used to compare the pore-forming activity of PorB. MOMP porin activity is detected using only $1 \mu \mathrm{g}$ protein (total outer-membrane protein) whilst 6-10 $\mu \mathrm{g}$ purified PorB is needed to observe comparable porin activity (Kubo \& Stephens, 2000). This suggests that PorB is much less efficient as a general porin or that the 


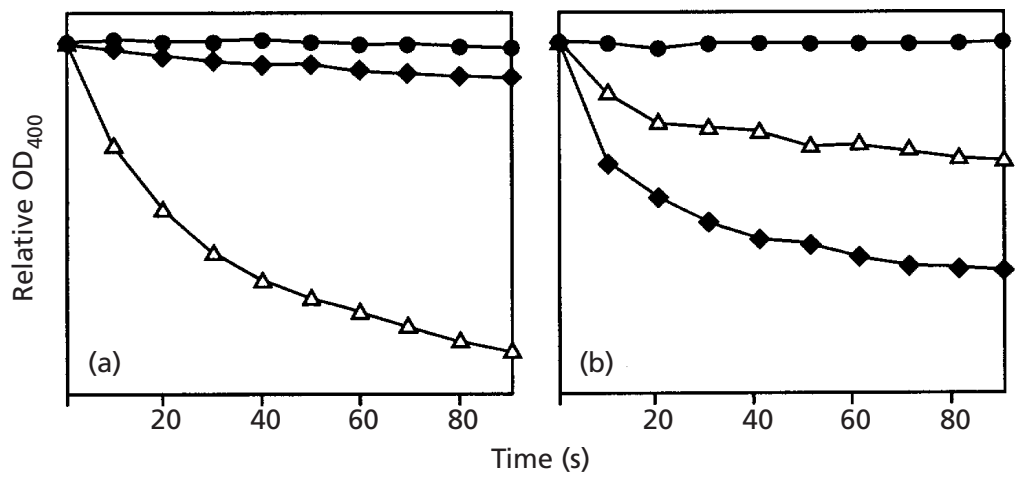

Fig. 1. Liposome-swelling analysis of PorB (a) and the outer membrane of $E$. coli expressing MOMP (OmpA) (b). Isotonic solutions of stachyose ( $)$, arabinose $(\bullet)$ and 2-oxoglutarate $(\triangle)$. The $y$-axis represents a range of $\mathrm{OD}_{400} 0 \cdot 15$.

purification process may have resulted in a less functional protein.

To determine if PorB had specificity for any molecule(s), we studied the genome sequence to determine if the inferred biology of Chlamydia could provide an idea of which molecules Chlamydia might need to obtain from the host. This analysis provided a list of orthologues of transporters that are important in the translocation of solutes across the inner membrane, including amino acid, polysaccharide, oligopeptide and dicarboxylate transporters (Stephens et al., 1998). Previous analysis of MOMP porin activity showed that amino acids, monoand disaccharides and oligopeptides enter efficiently through MOMP (Jones et al., 2000). However, PorB did not allow for the efficient entry of either amino acids or polysaccharides (Kubo \& Stephens, 2000). The presence of an orthologue to an inner-membrane dicarboxylate transporter, and the fact that Chlamydia spp. appear to have a truncated TCA cycle, suggest that chlamydiae may require exogenous 2-oxoglutarate from the host cell. Therefore, we tested the hypothesis that dicarboxylates could enter through the chlamydial outer membrane by measuring 2-oxoglutarate diffusion through the two known porins, PorB and MOMP.

The liposome-swelling assay with PorB and MOMP showed that the diffusion of 2-oxoglutarate was more efficient through PorB than MOMP (Fig. 1). Several controls were employed to support the PorB-based diffusion data. No diffusion of 2-oxoglutarate was seen using liposomes without protein or when using liposomes containing another chlamydial outer-membrane protein $(\mathrm{Omp} 85)$ that is a conserved bacterial protein (data not shown). Chlamydial Omp85 was used as a control protein; it was cloned, expressed in E. coli and purified by the same method used to purify PorB. Lysates of E. coli not expressing PorB were treated the same way as E. coli expressing PorB and subjected to nickel column chromatography. The column eluate was used as a control in all the assays to verify that no E. coli contaminants were responsible for the porin activity observed. The presence of the His tag on PorB did not affect its ability to function as a porin but it is possible that the His tag could unexpectedly influence the diffusion of some of the substrates. However, the activity of PorB was dose-response dependent as increasing

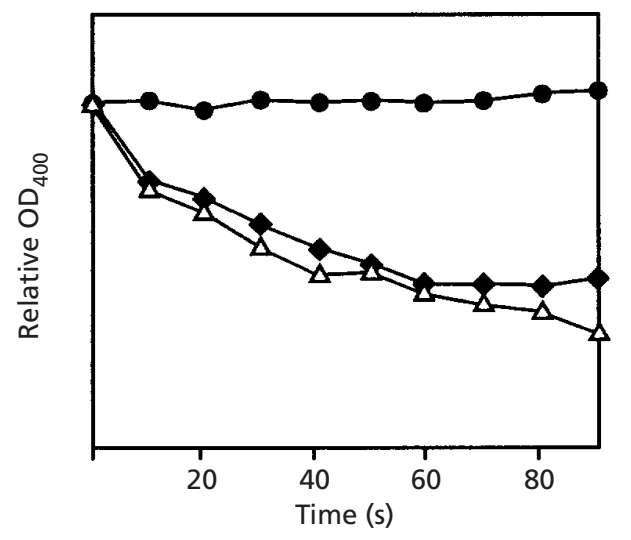

Fig. 2. Liposome-swelling assay to control for effects of ions that may be present in the test solute. Liposomes containing $\mathrm{NAD}^{+}$, stachyose and imidazole-NAD were diluted in isotonic test solutions of citrate $(0)$, oxaloacetate $(\bullet)$, and 2oxoglutarate $(\triangle)$. The $y$-axis represents a range of $\mathrm{OD}_{400} 0 \cdot 15$.

amounts of PorB added to liposomes resulted in increased swelling rates (data not shown).

One concern with the liposome assays was the possible influence of ions present in anionic solutes, such as 2oxoglutarate, that may cause ion fluxes potentially confounding the results of the assay. A liposome assay to control for the possibility of ion fluxes (Nikaido \& Rosenberg, 1983) was used to confirm the swelling assay results. Liposomes were made with $\mathrm{NAD}^{+}$-imidazole and stachyose to counteract any ion fluxes that may result from the presence of contaminating ions in the 2oxoglutarate solute used for the assay. This assay confirmed that the results in the initial liposome assays were not the result of ion fluxes and that oxaloacetate also entered efficiently through PorB whereas citrate did not enter (Fig. 2).

To further show that the 2-oxoglutarate was entering through PorB, we used an enzyme-linked liposome assay. The liposomes were made with 2-oxoglutarate dehydrogenase and $\mathrm{NAD}^{+}$inside and washed. The substrate, 2-oxoglutarate, was added to the outside of the liposomes and then the liposomes were measured for the formation of NADH by the increase in the $A_{340}$. The 


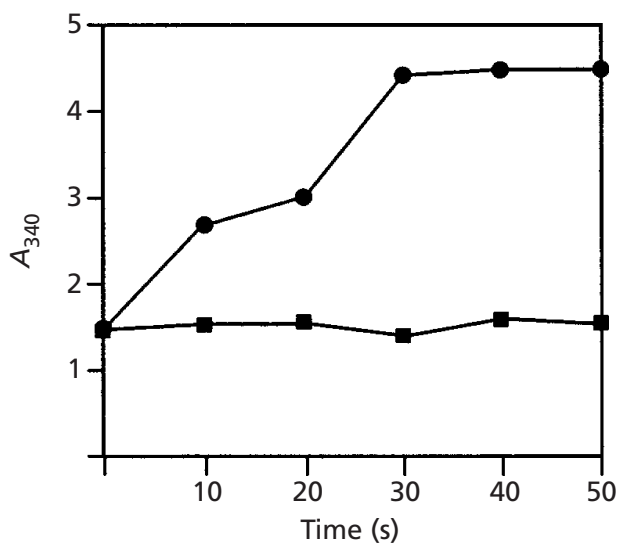

Fig. 3. Enzyme-linked liposome assay testing the entry and oxidation of 2-oxoglutarate. Liposomes were formulated with 2-oxoglutarate dehydrogenase and $\mathrm{NAD}^{+}$inside the vesicles and the substrate (2-oxoglutarate) was added to the outside. The formation of NADH using liposomes containing PorB (0) or lacking PorB $(\boldsymbol{\square})$ was measured by an increase in $A_{340}$.

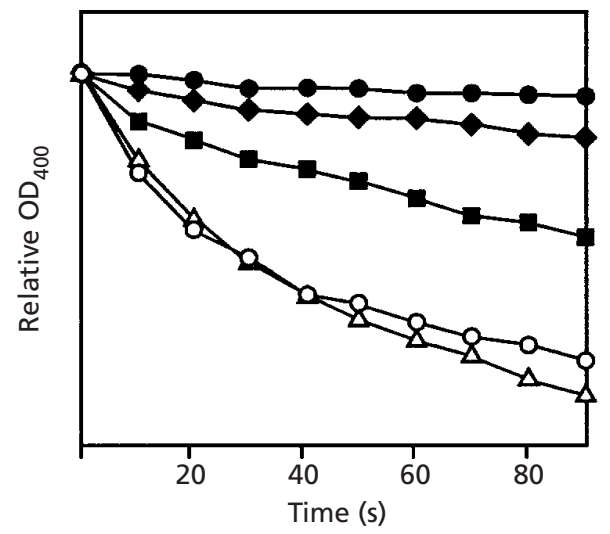

Fig. 4. Liposome-swelling analysis of PorB using TCA-cycle intermediates. Isotonic sugar solutions of stachyose (ひ), arabinose $(\diamond)$ and 2-oxoglutarate $(\triangle)$, malate $(\boldsymbol{\square})$ and succinate $(O)$. The $y$-axis represents a range of $\mathrm{OD}_{400} 0 \cdot 15$. result was the formation of NADH from 2-oxoglutarate that entered only in liposomes containing PorB (Fig. 3).

\section{TCA cycle molecules enter through PorB}

Based upon the finding that 2-oxoglutarate efficiently entered through PorB, a number of other TCA cycle intermediates were tested to assess whether this porin was specific for the 2-oxoglutarate substrate. Succinate (and oxaloacetate, data not shown) entered through PorB with rates similar to 2-oxoglutarate; however, malate did not enter efficiently (Fig. 4). Citrate did not enter through PorB (data not shown).

\section{Permeability specificity studies with PorB}

Since dicarboxylates of the TCA cycle were tested and diffused through PorB, we studied other molecular analogues to determine the capability of PorB to distinguish between related molecules (Fig. 5). A difference in carbon-chain lengths represented by adipate, glutarate, succinate and malonate did not show marked differences in diffusion compared to 2oxoglutarate, although 6-carbon adipate and 3-carbon malonate entered through PorB at slightly slower rates (data not shown). Thus PorB did not discriminate between different substrate chain lengths. The effects of small side groups were tested using analogues that differed only by specific side groups. For example, 2oxoglutarate and glutarate entered through PorB efficiently, but glutamate, which is similar in structure, did not. The presence of the amino group seems to retard the diffusion of glutamate and this likely explains why other amino acids do not enter into PorB efficiently (Kubo \& Stephens, 2000). A comparison of the 5-carbon compounds citrate and aconitate showed that only the presence of a hydroxyl group in citrate was sufficient to prevent the entry of citrate through PorB. Four-carbon malate and succinate also differ by the presence of a hydroxyl group and the diffusion rate was retarded for malate. Therefore, PorB can discern between very similar compounds, suggesting it is a substrate-specific

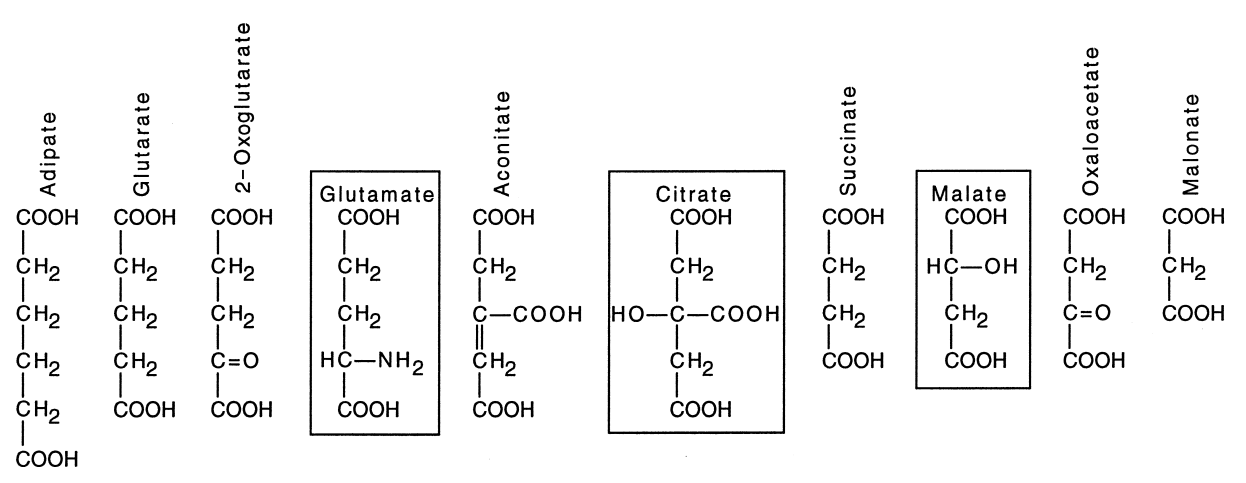

Fig. 5. Structures of compounds tested for diffusion into liposomes containing PorB. Compounds in boxes were not efficiently transported by PorB. 
porin. Our findings show that PorB facilitates the diffusion of 2-oxoglutarate and other select dicarboxylates to enter chlamydial outer membranes efficiently.

\section{DISCUSSION}

Unlike most other bacteria, growth of chlamydiae is limited to a single environmental niche, called an inclusion, within the eukaryotic cell vacuole. The nature of the inclusion as well as how nutrients enter into this environment has yet to be determined. Chlamydia spp. must obtain nutrients from their eukaryotic host cell by moving compounds across the vacuolar membrane and then across the chlamydial outer envelope. It has been reported that the vacuolar membrane is not permeable to molecules larger than $520 \mathrm{Da}$ and it has been proposed that nutrients must be actively transported (Heinzen $\&$ Hackstadt, 1997). Once inside the vacuole, a concentration gradient could drive the transport of some smaller compounds into Chlamydia through pores such as MOMP and PorB.

MOMP functions as a general porin that allows for the diffusion of various types of molecules within a size limit through the outer membrane, and those necessary for Chlamydia will be transported through the inner membrane by transporters. PorB is present in much smaller amounts than MOMP (Kubo \& Stephens, 2000) and likely has a more specific role than MOMP to allow for particular classes of molecules to be transported at a faster rate, much like substrate-specific porins found in other bacteria (Koebnik et al., 2000). Since chlamydiae rely on the host for much of the nutrients they need to grow, it is not surprising that they have substratespecific porins for the efficient transport of essential nutrients. PorB may be present to ensure that chlamydiae acquire 2-oxoglutarate to provide necessary metabolic intermediates and energy. Gene transcription and protein analyses have shown that por $B$ is not developmentally regulated (Kubo \& Stephens, 2000) and other genes such as transporters for glutamate $(g l t T)$ and glucose $(u h p C)$ also are not developmentally regulated (Iliffe-Lee \& McClarty, 2000). Thus Chlamydia is unlike most other bacteria that respond to environmental change by altering gene expression of porins and transporters. This may be because chlamydiae only grow in a singular environment within a host-cell vacuole; however, it is unknown how in vitro cultures may differ from in vivo infection in terms of the availability of carbon sources for chlamydial growth.

After diffusion through the outer membrane, there are many inner-membrane transporters that actively transport molecules into the cytoplasm of Chlamydia. The presence of a gene encoding a dicarboxylate transporter orthologue (SodTi) in Chlamydia suggests that 2oxoglutarate is transported across the inner membrane. The SodTi transporter in chloroplasts functions as a translocator that brings 2-oxoglutarate into the stroma in exchange for malate (Weber et al., 1995). Therefore, the SodTi orthologue in Chlamydia may function in much the same way by transporting 2-oxoglutarate through the inner membrane into the chlamydial cytosol. Once 2-oxoglutarate is transported through the inner membrane, it can then be used in the chlamydial TCA loop since chlamydiae apparently do not have the capability of producing 2-oxoglutarate and it has recently been shown that chlamydiae may utilize 2oxoglutarate as a carbon source to support growth (Iliffe-Lee \& McClarty, 2000).

PorB is the only porin in bacteria to show efficient diffusion for select dicarboxylates and is the second porin found in Chlamydia. PorB has a different function from MOMP and PorB can distinguish between similar molecules. However, the substrate selectivity determined for PorB is not biologically unique since a peroxisome porin also has specificity for dicarboxylates (Reumann et al., 1998). Peroxisomes are found in all but the most primitive eukaryotic cells and, like Chlamydia, they have access to intracellular substrates. Like PorB, the peroxisome porin does not form a general diffusion pore, but has particular properties analogous to specific and inducible porins that have been characterized in some Gram-negative bacteria (Koebnik et al., 2000; Luckey \& Nikaido, 1980). Although PorB and the peroxisome porin have analogous functions, sequence comparisons as well as secondary structure comparisons did not reveal significant similarity.

Investigation of the porin function of MOMP and PorB has allowed for a general understanding of the permeability of the outer membrane of Chlamydia and its relevance to the unique metabolic machinery as inferred by the genome sequence analysis. MOMP facilitates the efficient diffusion of polysaccharides (i.e. glucose) and amino acids including glutamate (Jones et al., 2000) that are then transported across the inner membrane by UhpC and GltT, respectively (Stephens et al., 1998). In contrast, PorB facilitates the efficient diffusion of dicarboxylates (i.e. 2-oxoglutarate) that are transported across the inner membrane by SodTi. Thus, PorB has an important role in providing TCA cycle intermediates that also produce cofactors such as NADH for the respiratory chain (McClarty, 1999).

\section{ACKNOWLEDGEMENTS}

We are very grateful to Dr H. Nikaido for valuable advice and discussion concerning liposome-swelling assays. This work was supported by NIH grants AI40250, AI39258, AI42156 and in part by the Grossman Doctoral Research Award.

\section{REFERENCES}

Bavoil, P., Ohlin, A. \& Schachter, J. (1984). Role of disulfide bonding in outer membrane structure and permeability in Chlamydia trachomatis. Infect Immun 44, 479-485.

Harper, A., Pogson, C. I., Jones, M. L. \& Pearce, J. H. (2000). Chlamydial development is adversely affected by minor changes in amino acid supply, blood plasma amino acid levels, and glucose deprivation. Infect Immun 68, 1457-1464.

Heinzen, R. A. \& Hackstadt, T. (1997). The Chlamydia trachomatis parasitophorous vacuolar membrane is not passively permeable 
to low-molecular-weight compounds. Infect Immun $\mathbf{6 5}$, 1088-1094.

Iliffe-Lee, E. R. \& McClarty, G. (2000). Regulation of carbon metabolism in Chlamydia trachomatis. Mol Microbiol 38, 20-30.

Jones, H. M., Kubo, A. \& Stephens, R. S. (2000). Design, expression and functional characterization of a synthetic gene encoding the Chlamydia trachomatis major outer membrane protein. Gene 258, 173-181.

Kalman, S., Mitchell, W., Marathe, R. \& 7 other authors (1999). Comparative genomes of Chlamydia pneumoniae and C. trachomatis. Nat Genet 21, 385-389.

Koebnik, R., Locher, K. P. \& Van Gelder, P. (2000). Structure and function of bacterial outer membrane proteins: barrels in a nutshell. Mol Microbiol 37, 239-253.

Kubo, A. \& Stephens, R. S. (2000). Characterization and functional analysis of PorB, a Chlamydia porin and neutralizing target. Mol Microbiol 38, 772-780.

Luckey, M. \& Nikaido, H. (1980). Specificity of diffusion channels produced by lambda phage receptor protein of Escherichia coli. Proc Natl Acad Sci US A 77, 167-171.

McClarty (1999). Chlamydial metabolism as inferred from the complete genome sequence. In Chlamydia: Intracellular Biology, Pathogenesis, and Immunity, pp. 69-100. Edited by R.S. Stephens. Washington, DC: American Society for Microbiology.
Moulder, J. W. (1991). Interaction of chlamydiae and host cells in vitro. Microbiol Rev 55, 143-190.

Nikaido, H. \& Rosenberg, E. Y. (1983). Porin channels in Escherichia coli: studies with liposomes reconstituted from purified proteins. J Bacteriol 153, 241-252.

Osborn, M. J. \& Munson, R. (1974). Separation of the inner (cytoplasmic) and outer membranes of gram-negative bacteria. Methods Enzymol 31, 642-653.

Reumann, S., Maier, E., Heldt, H. W. \& Benz, R. (1998). Permeability properties of the porin of spinach leaf peroxisomes. Eur J Biochem 251, 359-366.

Stephens, R. S., Kalman, S., Lammel, C. \& 9 other authors (1998). Genome sequence of an obligate intracellular pathogen of humans: Chlamydia trachomatis. Science 282, 754-759.

Weber, A., Menzlaff, E., Arbinger, B., Gutensohn, M., Eckerskorn, C. \& Flügge, U. I. (1995). The 2-oxoglutarate/malate translocator of chloroplast envelope membranes: molecular cloning of a transporter containing a 12-helix motif and expression of the functional protein in yeast cells. Biochemistry 34, 2621-2627.

Wyllie, S., Ashley, R. H., Longbottom, D. \& Herring, A. J. (1998). The major outer membrane protein of Chlamydia psittaci functions as a porin-like ion channel. Infect Immun 66, 5202-5207.

Received 19 April 2001; revised 26 June 2001; accepted 23 July 2001. 